\title{
Boundary form factors in finite volume
}

\author{
M. Kormos ${ }^{1 *}$ and G. Takács ${ }^{2 \dagger}$ \\ ${ }^{1}$ Institute for Theoretical Physics \\ Eötvös University, Budapest \\ and \\ ${ }^{2}$ HAS Research Group for Theoretical Physics \\ H-1117 Budapest, Pázmány Péter sétány 1/A.
}

7th April 2008

\begin{abstract}
We describe the volume dependence of matrix elements of local boundary fields to all orders in inverse powers of the volume. Using the scaling boundary Lee-Yang model as testing ground, we compare the matrix elements extracted from boundary truncated conformal space approach to exact form factors obtained using the bootstrap method. We obtain solid confirmation for the boundary form factor bootstrap, which is different from all previously available tests in that it is a non-perturbative and direct comparison of exact form factors to multi-particle matrix elements of local operators, computed from the Hamiltonian formulation of the quantum field theory.
\end{abstract}

\section{Introduction}

The investigation of integrable boundary quantum field theories started with the seminal work of Ghoshal and Zamolodchikov [1], who set up the boundary R-matrix bootstrap, which makes possible the determination of the reflection matrices and provides complete description of the theory on the mass shell.

For the calculation of correlation functions, matrix elements of local operators between asymptotic states have to be computed. In a boundary quantum field theory there are

\footnotetext{
${ }^{*}$ E-mail: kormos@general.elte.hu

†E-mail: takacs@elte.hu
} 
two types of operators, the bulk and the boundary operators, where their names indicate their localization point. The boundary form factor program for calculating the matrix elements of local boundary operators between asymptotic states was initiated in [2]. The validity of form factor solutions was checked in the case of the boundary scaling Lee-Yang model by calculating the two-point function using a spectral sum and comparing it to the prediction of conformal perturbation theory. In [3] the spectrum of independent form factor solutions in the scaling Lee-Yang model and the sinh-Gordon model was compared to the boundary operator content of the ultraviolet boundary conformal field theory and a complete agreement was found. Further solutions of the boundary form factor axioms were constructed and their structure was analyzed for the sinh-Gordon theory at the self-dual point in [4], and for the $A_{2}$ affine Toda field theory in [5].

While the checks performed so far have confirmed the validity of the boundary form factor bootstrap proposed in [2], they can still be considered incomplete. The spectral sum evaluated in that paper only receives a very small contribution from form factors with more than two particles, thus it cannot be considered as a test of higher form factor functions, and therefore it does not constitute a stringent verification of the singularity axioms, which form the basis of the recursive construction of form factors. The solution counting in [3] does involve form factors up to arbitrary number; however, the counting procedure uses only some rough features of the axioms such as power counting of the polynomials involved, and the structure of the kernels of the recursion relations.

It is therefore desirable to have a direct comparison of form factors to matrix elements of local operators evaluated directly from the boundary quantum field theory in a nonperturbative framework. For periodic boundary conditions, it was shown recently in [6, 7] that such a comparison can be achieved by extracting the matrix elements in finite volume, for which one can use the very efficient truncated conformal space approach. In this paper we give the extension of this framework to the boundary case, using the boundary scaling Lee-Yang model as our paradigmatic example.

The paper is structured as follows. In section 2 we review the necessary ingredients of the boundary form factor bootstrap and give the form factor solution in the boundary scaling Lee-Yang model for the boundary operator with the lowest scaling dimension, up to 6 particles. In section 3 we derive a framework which makes it possible to calculate finite volume matrix elements using the infinite volume form factors, to all orders in inverse powers of the volume $L$, i.e. neglecting only corrections that decay exponentially fast with $L$. In section 4 we give the necessary background on the boundary truncated conformal space method, and also discuss the numerical precision and sources of errors. The actual comparison between the predictions from the bootstrap solution and the numerically evaluated finite volume matrix elements is performed in section 5, and the conclusions are drawn in section 6 . 


\section{Boundary form factor bootstrap for the scaling Lee- Yang model}

\subsection{The boundary form factor axioms}

The axioms satisfied by the form factors of a local boundary operator were derived in [2]. Here we only list them without much further explanation. Let us suppose that we treat an integrable boundary quantum field theory in the (infinite volume) domain $x<0$, with a single scalar particle of mass $m$, which has a two-particle $S$ matrix $S(\theta)$ (using the standard rapidity parametrization) and a one-particle reflection factor $R(\theta)$ off the boundary, satisfying the boundary reflection factor bootstrap conditions of Ghoshal and Zamolodchikov [1]. For a local operator $\mathcal{O}(t)$ localized at the boundary (located at $x=0$, and parametrized by the time coordinate $t$ ) the form factors are defined as

$$
\begin{aligned}
{ }_{\text {out }}\left\langle\theta_{1}^{\prime}, \theta_{2}^{\prime}, \ldots, \theta_{m}^{\prime}|\mathcal{O}(t)| \theta_{1}, \theta_{2}, \ldots,\right. & \left.\theta_{n}\right\rangle_{\text {in }} \\
& F_{m n}^{\mathcal{O}}\left(\theta_{1}^{\prime}, \theta_{2}^{\prime}, \ldots, \theta_{m}^{\prime} ; \theta_{1}, \theta_{2}, \ldots, \theta_{n}\right) e^{-i m t\left(\sum \cosh \theta_{i}-\sum \cosh \theta_{j}^{\prime}\right)}
\end{aligned}
$$

for $\theta_{1}>\theta_{2}>\ldots>\theta_{n}>0$ and $\theta_{1}^{\prime}<\theta_{2}^{\prime}<\ldots<\theta_{m}^{\prime}<0$, using the asymptotic in/out state formalism introduced in [8]. They can be extended analytically to complex values of the rapidity variables. With the help of the crossing relations derived in [2] all form factors can be expressed in terms of the elementary form factors

$$
{ }_{\text {out }}\left\langle 0|\mathcal{O}(0)| \theta_{1}, \theta_{2}, \ldots, \theta_{n}\right\rangle_{\text {in }}=F_{n}^{\mathcal{O}}\left(\theta_{1}, \theta_{2}, \ldots, \theta_{n}\right)
$$

which can be shown to satisfy the following axioms ${ }^{1}$ :

I. Permutation:

$$
F_{n}^{\mathcal{O}}\left(\theta_{1}, \ldots, \theta_{i}, \theta_{i+1}, \ldots, \theta_{n}\right)=S\left(\theta_{i}-\theta_{i+1}\right) F_{n}^{\mathcal{O}}\left(\theta_{1}, \ldots, \theta_{i+1}, \theta_{i}, \ldots, \theta_{n}\right)
$$

II. Reflection:

$$
F_{n}^{\mathcal{O}}\left(\theta_{1}, \ldots, \theta_{n-1}, \theta_{n}\right)=R\left(\theta_{n}\right) F_{n}^{\mathcal{O}}\left(\theta_{1}, \ldots, \theta_{n-1},-\theta_{n}\right)
$$

III. Crossing reflection:

$$
F_{n}^{\mathcal{O}}\left(\theta_{1}, \theta_{2}, \ldots, \theta_{n}\right)=R\left(i \pi-\theta_{1}\right) F_{n}^{\mathcal{O}}\left(2 i \pi-\theta_{1}, \theta_{2}, \ldots, \theta_{n}\right)
$$

IV. Kinematical singularity

$$
-i \operatorname{Res}_{\theta=\theta^{\prime}} F_{n+2}^{\mathcal{O}}\left(\theta+i \pi, \theta^{\prime}, \theta_{1}, \ldots, \theta_{n}\right)=\left(1-\prod_{i=1}^{n} S\left(\theta-\theta_{i}\right) S\left(\theta+\theta_{i}\right)\right) F_{n}^{\mathcal{O}}\left(\theta_{1}, \ldots, \theta_{n}\right)
$$

\footnotetext{
${ }^{1}$ There is a further axiom corresponding to boundary excited state poles, but it will not be needed in the sequel.
} 
V. Boundary kinematical singularity

$$
-i \operatorname{Res}_{\theta=0} F_{n+1}^{\mathcal{O}}\left(\theta+\frac{i \pi}{2}, \theta_{1}, \ldots, \theta_{n}\right)=\frac{g}{2}\left(1-\prod_{i=1}^{n} S\left(\frac{i \pi}{2}-\theta_{i}\right)\right) F_{n}^{\mathcal{O}}\left(\theta_{1}, \ldots, \theta_{n}\right)
$$

where $g$ is the one-particle coupling to the boundary

$$
R(\theta) \sim \frac{i g^{2}}{2 \theta-i \pi} \quad, \quad \theta \sim i \frac{\pi}{2}
$$

VI. Bulk dynamical singularity

$$
-i \operatorname{Res}_{\theta=\theta^{\prime}} F_{n+2}^{\mathcal{O}}\left(\theta+i u, \theta^{\prime}-i u, \theta_{1}, \ldots, \theta_{n}\right)=\Gamma F_{n+1}^{\mathcal{O}}\left(\theta, \theta_{1}, \ldots, \theta_{n}\right)
$$

corresponding to a bound state pole of the $S$ matrix

$$
S(\theta) \sim \frac{i \Gamma^{2}}{\theta-2 i u} \quad, \quad \theta \sim 2 i u
$$

(in a theory with a single particle, the only possible value is $u=\pi / 3$ ).

We further assume maximum analyticity i.e. that the form factors have only the minimal singularity structure consistent with the above axioms. The general form factor solution can be written in the following form [2]

$$
F_{n}\left(\theta_{1}, \theta_{2}, \ldots, \theta_{n}\right)=G_{n}\left(\theta_{1}, \theta_{2}, \ldots, \theta_{n}\right) \prod_{i=1}^{n} r\left(\theta_{i}\right) \prod_{i<j} f\left(\theta_{i}-\theta_{j}\right) f\left(\theta_{i}+\theta_{j}\right)
$$

where $f$ is the minimal bulk two-particle form factor satisfying the conditions

$$
f(\theta)=S(\theta) f(-\theta), \quad f(i \pi+\theta)=f(i \pi-\theta)
$$

and having the minimum possible number of singularities in the physical strip $0 \leq \theta<\pi$ together with the slowest possible growth at infinity [9], and $r$ is the minimal boundary one-particle form factor satisfying

$$
r(\theta)=R(\theta) r(-\theta) \quad ; \quad r(i \pi+\theta)=R(-\theta) r(i \pi-\theta)
$$

plus analytic conditions similar to those of $f$, but in this case in the strip $0 \leq \theta<\pi / 2$.

The functions $G_{n}$ are totally symmetric and meromorphic in the rapidities $\theta_{i}$. They are also even and periodic in them with the period $2 \pi i$, so they can only be functions of the variables

$$
y_{i}=\mathrm{e}^{\theta_{i}}+\mathrm{e}^{-\theta_{i}}
$$

In a theory with only one particle (such as the scaling Lee-Yang model), the only possible singularity of the $S$ matrix in the physical strip is located at $\theta=2 \pi i / 3$ corresponding to 
the self-fusion of the particle (plus the crossed channel pole for the same process at $\pi i / 3$ ) and the relevant fusion coupling is defined as

$$
\Gamma^{2}=-i \operatorname{Res}_{\theta=\frac{2 \pi}{3} i} S(\theta)
$$

Assuming $f$ is chosen such that it has a pole at $\theta=2 \pi i / 3$ so that it encodes this singularity and that the boundary dynamical singularities (but not the kinematical ones!) are similarly contained in the $1 \mathrm{PFF}$ function $r$, the functions $G_{n}$ can be written in the form

$$
G_{n}\left(\theta_{1}, \theta_{2}, \ldots, \theta_{n}\right)=\frac{P_{n}\left(y_{1}, y_{2} \ldots, y_{n}\right)}{\prod_{i} y_{i} \prod_{i<j}\left(y_{i}+y_{j}\right)}
$$

where the $P_{n}$ are entire functions symmetric in their arguments. Assuming that the correlation functions involving the operators in consideration only have power-like short-distance singularities, the asymptotic growth of the form factors is limited by

$$
F_{n}\left(\theta_{1}+\Lambda, \theta_{2}+\Lambda, \ldots, \theta_{n}+\Lambda\right) \sim \mathrm{e}^{d \Lambda}
$$

for some real number $d$, and therefore $P_{n}$ can only be polynomials of finite degree [2].

\subsection{Scaling Lee-Yang model with boundary}

The scaling Lee-Yang model with boundary is a combined bulk and boundary perturbation of the boundary version of the $\mathcal{M}_{2,5}$ Virasoro minimal model, which was investigated in detail in [10]. The conformal field theory has central charge $c=-22 / 5$ and the Virasoro algebra has two irreducible representations $V_{\Delta}$ with highest weight $\Delta=\Delta_{1,1}=0$ and $\Delta=\Delta_{1,2}=-1 / 5$. There is a unique nontrivial relevant bulk perturbation given by the spinless field $\phi$ with scaling dimensions $\Delta=\bar{\Delta}=-1 / 5$ which for an appropriate choice of the sign of the coupling flows to a massive infrared fixed point.

Boundary conformal field theory was developed in $[11,12,13]$ and the interested reader is referred to them for details. Applying the formalism to the conformal Lee-Yang model it can be seen that there are two conformally invariant boundary conditions. On one of them, denoted 1 (i.e. identity) in [10], there is no possibility for a boundary perturbation. In the other case, denoted $\Phi$ in [10], there is a nontrivial relevant boundary field $\varphi$ with scaling dimension $-1 / 5$ and the general perturbed boundary conformal field theory action can be written as

$$
\mathcal{A}_{\lambda, \Phi(h)}=\mathcal{A}_{\Phi}+\lambda \int_{-\infty}^{\infty} d y \int_{-\infty}^{0} d x \phi(x, y)+h \int_{-\infty}^{\infty} d y \varphi(y)
$$

where $\mathcal{A}_{\Phi}$ denotes the action for $\mathcal{M}(2 / 5)$ with the $\Phi$ boundary condition imposed at $x=0$, and $\lambda$ and $h$ denote the bulk and boundary couplings respectively. The action of $\mathcal{A}_{\lambda, 1}$ is similar, but the last term on the right hand side is missing. For $\lambda>0$ the bulk behaviour 
is described by an integrable massive theory having only a single particle with mass $m$ with the following $\mathrm{S}$ matrix [14]:

$$
S(\theta)=-\left(\frac{1}{3}\right)\left(\frac{2}{3}\right)=-\left[\frac{1}{3}\right] \quad ; \quad(x)=\frac{\sinh \left(\frac{\theta}{2}+\frac{i \pi x}{2}\right)}{\sinh \left(\frac{\theta}{2}-\frac{i \pi x}{2}\right)} \quad, \quad[x]=(x)(1-x)
$$

The minimal bulk two-particle form factor only has a zero at $\theta=0$ and a pole at $\theta=\frac{2 \pi i}{3}$ in the strip $0 \leq \Im m(\theta)<\pi$ and is of the form [15]:

$$
f(\theta)=\frac{y-2}{y+1} v(i \pi-\theta) v(-i \pi+\theta) \quad, \quad y=e^{\theta}+e^{-\theta}
$$

where

$$
v(\theta)=\exp \left\{2 \int_{0}^{\infty} \frac{d t}{t} e^{i \frac{\theta t}{\pi}} \frac{\sinh \frac{t}{2} \sinh \frac{t}{3} \sinh \frac{t}{6}}{\sinh ^{2} t}\right\}
$$

For the perturbed $\Phi$ boundary, the reflection amplitude of the particle depends on the boundary coupling constant as [10]

$$
R(\theta)_{\Phi}=\left(\frac{1}{2}\right)\left(\frac{1}{6}\right)\left(-\frac{2}{3}\right)\left[\frac{b+1}{6}\right]\left[\frac{b-1}{6}\right]
$$

where the dimensionless parameter $b$ is related to the dimensionful $h$ as [16]

$$
\begin{gathered}
h(b)=-h_{\text {crit }} m^{6 / 5} \sin \left[\left(b+\frac{1}{2}\right) \frac{\pi}{5}\right] \\
h_{\text {crit }}=\pi^{\frac{3}{5}} 2^{\frac{4}{5}} 5^{\frac{1}{4}} \frac{\sin \frac{2 \pi}{5}}{\sqrt{\Gamma\left(\frac{3}{5}\right) \Gamma\left(\frac{4}{5}\right)}}\left(\frac{\Gamma\left(\frac{2}{3}\right)}{\Gamma\left(\frac{1}{6}\right)}\right)^{\frac{6}{5}}=0.685289983991 \ldots
\end{gathered}
$$

and $m$ is the mass of the particle giving the overall scale in the infrared description, which is related to the bulk coupling $\lambda$ as $[17,18]$

$$
\begin{aligned}
m= & \kappa \lambda^{5 / 12} \\
& \kappa=2^{19 / 12} \sqrt{\pi} \frac{(\Gamma(3 / 5) \Gamma(4 / 5))^{5 / 12}}{5^{5 / 16} \Gamma(2 / 3) \Gamma(5 / 6)}=2.64294466304 \ldots
\end{aligned}
$$

In the case of the $\mathbf{1}$ boundary the reflection amplitude is the parameter independent expression

$$
R(\theta)_{1}=\left(\frac{1}{2}\right)\left(\frac{1}{6}\right)\left(-\frac{2}{3}\right)
$$




\subsection{Recursion relations and form factor solution for the $\Phi$ bound- ary condition}

The minimal boundary one-particle form factor is [2]

$$
r_{\Phi}(\theta)=\frac{i \sinh \theta}{\left(\sinh \theta-i \sin \frac{\pi(b+1)}{6}\right)\left(\sinh \theta-i \sin \frac{\pi(b-1)}{6}\right)} u(\theta)
$$

where

$$
u(\theta)=\exp \left\{\int_{0}^{\infty} \frac{d t}{t}\left[\frac{1}{\sinh \frac{t}{2}}-2 \cosh \frac{t}{2} \cos \left[\left(\frac{i \pi}{2}-\theta\right) \frac{t}{\pi}\right] \frac{\sinh \frac{5 t}{6}+\sinh \frac{t}{2}-\sinh \frac{t}{3}}{\sinh ^{2} t}\right]\right\}
$$

Taking the following Ansatz

$$
F_{n}\left(\theta_{1}, \ldots, \theta_{n}\right)=N H_{n} Q_{n}\left(y_{1}, \ldots, y_{n}\right) \prod_{i} \frac{r_{\Phi}\left(\theta_{i}\right)}{y_{i}} \prod_{i<j} \frac{f\left(\theta_{i}-\theta_{j}\right) f\left(\theta_{i}+\theta_{j}\right)}{y_{i}+y_{j}}
$$

where

$$
H_{n}=\left(\frac{i 3^{\frac{1}{4}}}{2^{\frac{1}{2}} v(0)}\right)^{n}
$$

and $N$ is a normalization constant to be fixed later, the (2.8) bulk dynamical $(\mathcal{D}),(2.5)$ bulk

kinematical $(\mathcal{K})$ and $(2.6)$ boundary kinematical $(\mathcal{B})$ singularity axioms give the following recursion relations for the polynomials $Q_{n}[2,3]$

$\mathcal{D}: \quad Q_{2}\left(y_{+}, y_{-}\right)=\left(y^{2}-3+\beta\right) Q_{1}(y)$

$$
Q_{n+2}\left(y_{+}, y_{-}, y_{1}, \ldots, y_{n}\right)=\left(y^{2}-3+\beta\right) D_{n}\left(y \mid y_{1}, \ldots y_{n}\right) Q_{n+1}\left(y, y_{1}, \ldots, y_{n}\right) \quad, n>0
$$

$\mathcal{K}: \quad Q_{2}(-y, y)=0$

$$
Q_{n+2}\left(-y, y, y_{1}, \ldots, y_{n}\right)=\left(y^{4}-(3+\beta) y^{2}+\beta^{2}\right) K_{n}\left(y \mid y_{1}, \ldots y_{n}\right) Q_{n}\left(y_{1}, \ldots, y_{n}\right) \quad, n>0
$$

$\mathcal{B}: \quad Q_{1}(0)=0$

$$
Q_{n+1}\left(0, y_{1}, \ldots y_{n}\right)=\beta B_{n}\left(y_{1}, \ldots y_{n}\right) Q_{n}\left(y_{1}, \ldots, y_{n}\right) \quad, \quad n>0 \text {; }
$$

where we introduced the parameter

$$
\beta=1+2 \cos \frac{\pi b}{6}
$$

and $D_{n}, K_{n}$ and $B_{n}$ are given by

$$
\begin{gathered}
K_{n}\left(y \mid y_{1}, \ldots y_{n}\right)=\frac{1}{2\left(y_{+}-y_{-}\right)}\left[\prod_{i=1}^{n}\left(y_{i}-y_{-}\right)\left(y_{i}+y_{+}\right)-\prod_{i=1}^{n}\left(y_{i}+y_{-}\right)\left(y_{i}-y_{+}\right)\right] \\
B_{n}\left(y_{1}, \ldots, y_{n}\right)=\frac{1}{2 \sqrt{3}}\left(\prod_{i=1}^{n}\left(y_{i}+\sqrt{3}\right)-\prod_{i=1}^{n}\left(y_{i}-\sqrt{3}\right)\right)
\end{gathered}
$$




$$
D_{n}\left(y \mid y_{1}, \ldots y_{n}\right)=\prod_{i=1}^{n}\left(y+y_{i}\right)
$$

where

$$
\begin{aligned}
& y_{+}=\omega z+\omega^{-1} z^{-1} \\
& y_{-}=\omega^{-1} z+\omega z^{-1} \quad, \quad \omega=\mathrm{e}^{\frac{i \pi}{3}}
\end{aligned}
$$

with the auxiliary variable $z$ defined as a solution of $y=z+z^{-1}$ (i.e. writing $y=2 \cosh \theta$ we obtain $z=\mathrm{e}^{\theta}$ ). The symmetry of the above expressions in $y_{ \pm}$ensures that the resulting relations only depend on $y$ and also that $K_{n}$ is a polynomial in all of its variables [3].

The polynomials $Q_{n}$ can be expanded using the elementary symmetric polynomials defined by the generating function

$$
\prod_{i=1}^{n}\left(z+y_{i}\right)=\sum_{k=0}^{n} z^{n-k} \sigma_{k}\left(y_{1}, \ldots, y_{n}\right)
$$

The solution of the recursion relations $\mathcal{D}, \mathcal{K}$ and $\mathcal{B}$ proceeds as follows. All the recursion coefficients $D_{n}, K_{n}$ and $B_{n}$ can be expanded in products of symmetric polynomials of the variables $y_{1}, \ldots, y_{n}$ and, for the first two, powers of the additional variable $y$. For the minimal solution of these equations the polynomials $Q_{n}$ have degree $n(n+1) / 2$ [2], so we can write an Ansatz as a general linear combination of products of elementary symmetric polynomials not exceeding this degree. Using the relation

$$
\sigma_{k}\left(y, y_{1}, \ldots, y_{n}\right)=\sigma_{k}\left(y_{1}, \ldots, y_{n}\right)+y \sigma_{k-1}\left(y_{1}, \ldots, y_{n}\right)
$$

we can expand both sides of the recursion relations in terms of products of $\sigma_{k}$ and powers of $y$. Equating the coefficients of independent terms on the two sides, we obtain a linear system of equations for the coefficients of the Ansatz, which can then be solved. The minimal solution turns out to be unique (apart from an overall normalization that can be fixed by choosing the coefficient of $Q_{1}$ ), and up to 6 particles it is given by

$$
\begin{aligned}
Q_{1}^{\varphi}= & \sigma_{1} \\
Q_{2}^{\varphi}= & \sigma_{1}\left(\beta+\sigma_{2}\right) \\
Q_{3}^{\varphi}= & \sigma_{1}\left[\sigma_{3}\left(\sigma_{2}+3\right)+\beta \sigma_{1}\left(\sigma_{2}+\beta\right)\right] \\
Q_{4}^{\varphi}= & \sigma_{1}\left(\sigma_{2}+3\right)\left[\left(3 \sigma_{1}+\sigma_{3}\right) \sigma_{4}+\beta\left(\sigma_{2}+3\right) \sigma_{3}+\beta^{2} \sigma_{1}\left(\sigma_{2}+\beta\right)\right] \\
Q_{5}^{\varphi}= & \sigma_{1}\left(\left(3+\sigma_{2}\right)\left(3 \sigma_{1}+\sigma_{3}\right)-\sigma_{5}\right) \\
& \times\left[\sigma_{4}\left(3 \beta \sigma_{1}+\beta \sigma_{3}+\sigma_{5}\right)+(\beta-3) \beta\left(\beta^{2} \sigma_{1}-\sigma_{5}\right)+\left(3+\sigma_{2}\right)\left(\beta^{3} \sigma_{1}+\beta^{2} \sigma_{3}+3 \sigma_{5}\right)\right] \\
Q_{6}^{\varphi}= & \sigma_{1}\left[-\sigma_{4} \sigma_{5}-3 \sigma_{1} \sigma_{6}+\left(\sigma_{2}+3\right)\left(3 \sigma_{1} \sigma_{4}+\sigma_{3} \sigma_{4}-3 \sigma_{5}-\sigma_{1} \sigma_{6}\right)+3\left(\sigma_{2}+3\right)^{2}\left(3 \sigma_{1}+\sigma_{3}\right)\right] \\
& \times\left\{\beta\left(\sigma_{2}+3\right)\left(\beta^{3} \sigma_{1}+\beta^{2} \sigma_{3}+3 \sigma_{5}\right)+\left(9 \sigma_{1}+3 \sigma_{3}+\sigma_{5}\right)\left(3 \sigma_{4}+\sigma_{6}\right)\right. \\
& +(\beta-3)\left[81 \sigma_{1}-9 \sigma_{5}+\sigma_{4} \sigma_{5}+3 \sigma_{1} \sigma_{6}\right. \\
& \left.\left.+(\beta+3)\left(3 \sigma_{1} \sigma_{4}+\sigma_{3} \sigma_{4}-\sigma_{1} \sigma_{6}+(\beta-3)\left(9 \sigma_{1}+\beta^{2} \sigma_{1}-\sigma_{5}\right)\right)\right]\right\}
\end{aligned}
$$


The first three form factors in this sequence were originally computed in [2] where it was demonstrated that this solution corresponds to the relevant boundary field $\varphi$, which gives the boundary perturbation according to (2.11). The complete form factors can be written as [2]

$$
F_{n}\left(\theta_{1}, \ldots, \theta_{n}\right)=\langle\varphi\rangle H_{n} Q_{n}^{\varphi}\left(y_{1}, \ldots, y_{n}\right) \prod_{i} \frac{r_{\Phi}\left(\theta_{i}\right)}{y_{i}} \prod_{i<j} \frac{f\left(\theta_{i}-\theta_{j}\right) f\left(\theta_{i}+\theta_{j}\right)}{y_{i}+y_{j}}
$$

where

$$
\langle\varphi\rangle=-\frac{5}{6 h_{\text {crit }}} \frac{\cos \left(\frac{\pi b}{6}\right)}{\cos \left(\frac{\pi}{10}(2 b+1)\right)} m^{-1 / 5}
$$

is the exact expectation value of the boundary operator $\varphi[10]$.

\section{Boundary form factors in finite volume}

\subsection{Bethe-Yang equations}

Let us consider an integrable boundary quantum field theory with particles of species $a=1, \ldots, N$ and corresponding masses $m_{a}$. As usual in two-dimensional field theory, we label asymptotic particles with their rapidities $\theta$, which gives their energy and momentum as

$$
E_{a} \pm p_{a}=m_{a} \mathrm{e}^{ \pm \theta_{a}}
$$

We assume the bulk scattering is diagonal and is given by the two-particle $S$ matrices

$$
S_{a_{1} a_{2}}\left(\theta_{1}-\theta_{2}\right)=\mathrm{e}^{i \delta_{a_{1} a_{2}}\left(\theta_{1}-\theta_{2}\right)}
$$

We further assume that the reflection on the boundaries is also diagonal and is given by the reflection factors

$$
R_{a}^{(\alpha)}(\theta)=\mathrm{e}^{i \delta_{a}^{(\alpha)}(\theta)} \quad, \quad R_{a}^{(\beta)}(\theta)=\mathrm{e}^{i \delta_{a}^{(\beta)}(\theta)}
$$

where $\alpha$ and $\beta$ denote the left and right boundary conditions, respectively.

In the diagonal case, the multi-particle energy levels in a finite volume $L$ are described by the following Bethe-Yang equations [19]:

$$
Q_{j}\left(\theta_{1}, \ldots, \theta_{n}\right)_{a_{1} \ldots a_{n}}=2 \pi I_{j}
$$

where the phases describing the wave function monodromy are given by

$Q_{j}\left(\theta_{1}, \ldots, \theta_{n}\right)_{a_{1} \ldots a_{n}}=2 m_{a_{j}} L \sinh \theta_{j}+\sum_{k \neq j}\left(\delta_{a_{j} a_{k}}\left(\theta_{j}-\theta_{k}\right)+\delta_{a_{j} a_{k}}\left(\theta_{j}+\theta_{k}\right)\right)+\delta_{a_{j}}^{(\alpha)}\left(\theta_{j}\right)+\delta_{a_{j}}^{(\beta)}\left(\theta_{j}\right)$

Here all rapidities $\theta_{j}$ (and accordingly all quantum numbers $I_{j}$ ) are taken to be positive ${ }^{2}$. We can take the quantum numbers ordered as $I_{1} \leq \ldots \leq I_{n}$; in the scaling Lee-Yang model

\footnotetext{
${ }^{2}$ Boundary reflections change the sign of the momentum, so finite volume multi-particle states can be characterized by the absolute value of the rapidities.
} 
they must all be different due to the exclusion principle. We denote the corresponding multi-particle state by

$$
\left|\left\{I_{1}, \ldots, I_{n}\right\}\right\rangle_{a_{1} \ldots a_{n}, L}
$$

and its energy (relative to the ground state) is given by

$$
E_{I_{1} \ldots I_{n}}(L)=\sum_{j=1}^{n} m_{a_{j}} \cosh \tilde{\theta}_{j}
$$

where $\left\{\tilde{\theta}_{j}\right\}_{j=1, \ldots, n}$ is the solution of eqns. (3.2) at the given volume $L$. The Bethe-Yang equations gives the energy of the multi-particle states to all order in $1 / L$, neglecting only finite size effects decaying exponentially with $L$.

\subsection{Non-diagonal matrix elements}

Following the ideas outlined in $[6,7]$ we begin with examining a two-point function of two boundary operators

$$
\left\langle\mathcal{O}_{1}(\tau) \mathcal{O}_{2}(0)\right\rangle_{L}
$$

where $\tau$ is Euclidean time. Let us suppose for definiteness that we consider operators located on the right boundary.

We first need to establish that the finite size corrections to the two-point function will then be of the form

$$
\left\langle\mathcal{O}_{1}(\tau) \mathcal{O}_{2}(0)\right\rangle_{L}-\left\langle\mathcal{O}_{1}(\tau) \mathcal{O}_{2}(0)\right\rangle \sim \mathrm{e}^{-\mu L}
$$

Unfortunately, for the boundary situation there is no analogue of Lüscher's systematic finite volume expansion [20] that was used for periodic boundary conditions. Let us recall that the essential input in the argument is the analytic structure of Green's function, which was worked out for the boundary case in [8]; just as in the bulk case, the singularities are given by diagrams with all internal lines on-shell. It can be seen from Lüscher's derivation that the correction results from the singularities of momentum space Green's functions which occur in the momentum integrals of the finite volume expansion, and the exponent $\mu$ (which is on the scale of the mass gap of the theory) is given by the singularity lying closest to the physical domain. This argument cannot be considered a proper derivation; it would go much beyond the scope of this paper and requires developing the finite volume expansion for the boundary case. However, we still have a strong reason to accept that (3.3) holds; in retrospect, this is also confirmed by the numerical results in section 5 .

Using (3.3) we can then follow the arguments outlined in [6] without essential modifications. Just as in the periodic case we find that the infinite volume and finite volume matrix elements are just related by the square root of the ratio of normalization of the corresponding states (up to the corrections neglected in (3.3)). This results in the following relation:

$$
\left\langle 0|\mathcal{O}(0)|\left\{I_{1}, \ldots, I_{n}\right\}\right\rangle_{a_{1} \ldots a_{n}, L}=\frac{F_{a_{1} \ldots a_{n}}^{\mathcal{O}}\left(\tilde{\theta}_{1}, \ldots, \tilde{\theta}_{n}\right)}{\sqrt{\rho_{a_{1} \ldots a_{n}}\left(\tilde{\theta}_{1}, \ldots, \tilde{\theta}_{n}\right)}}+O\left(\mathrm{e}^{-\mu L}\right)
$$


where $F_{a_{1} \ldots a_{n}}^{\mathcal{O}}\left(\tilde{\theta}_{1}, \ldots, \tilde{\theta}_{n}\right)$ is the form factor of the operator $\mathcal{O}$ (in the infinite volume theory, i.e. on the half-line $x<0),\left\{\tilde{\theta}_{j}\right\}_{j=1, \ldots, n}$ is the solution of eqns. (3.2) at the given volume $L$, and

$$
\rho_{a_{1} \ldots a_{n}}\left(\tilde{\theta}_{1}, \ldots, \tilde{\theta}_{n}\right)=\operatorname{det}\left\{\frac{\partial Q_{k}\left(\theta_{1}, \ldots, \theta_{n}\right)_{a_{1} \ldots a_{n}}}{\partial \theta_{l}}\right\}_{k, l=1, \ldots, n}
$$

is the finite volume density of states, which is the Jacobi determinant of the mapping between the space of quantum numbers and the space of rapidities given by the Bethe-Yang equations (3.2). Using the crossing relations derived in [2] and following the arguments of [6], general matrix elements can be written as

$$
\begin{gathered}
b_{1} \ldots b_{m}\left\langle\left\{\left\{I_{1}^{\prime}, \ldots, I_{m}^{\prime}\right\}|\mathcal{O}(0)|\left\{I_{1}, \ldots, I_{n}\right\}\right\rangle_{a_{1} \ldots a_{n}, L}=\right. \\
\frac{F_{\bar{b}_{m} \ldots \bar{b}_{1} a_{1} \ldots a_{n}}\left(\tilde{\theta}_{m}^{\prime}+i \pi, \ldots, \tilde{\theta}_{1}^{\prime}+i \pi, \tilde{\theta}_{1}, \ldots, \tilde{\theta}_{n}\right)}{\sqrt{\rho_{a_{1} \ldots a_{n}}\left(\tilde{\theta}_{1}, \ldots, \tilde{\theta}_{n}\right) \rho_{b_{1} \ldots b_{m}}\left(\tilde{\theta}_{1}^{\prime}, \ldots, \tilde{\theta}_{m}^{\prime}\right)}}+O\left(\mathrm{e}^{-\mu L}\right)
\end{gathered}
$$

as long as the sets of the rapidities corresponding to the two states, $\left\{\tilde{\theta}_{j}\right\}_{j=1, \ldots, n}$ and $\left\{\tilde{\theta}_{j}^{\prime}\right\}_{j=1, \ldots, m}$, are disjoint i.e. when there are no disconnected contributions. Here $\bar{b}_{j}$ benotes the particle species conjugate to $b_{j}$.

The exponent $\mu$ in $(3.4,3.6)$ can also be estimated using the Poisson summation formula. The argument for the periodic case was written down in subsection 2.2 of ref. [6] and carries over without essential modification. The result is that the deviation between the discrete and continuous versions of the spectral sums is determined by the analytic singularity structure of the form factors and the phase-shifts involved in $(3.4,3.6)$ and the leading correction is given by the one with the smallest imaginary part in rapidity variables, just as the correction indicated in (3.3). Taking the two sources of exponential corrections together the result is that it is the singularity closest to the physical domain which determines the leading exponential correction in $(3.4,3.6)$. In fact this was already tacitly assumed by using the same exponent $\mu$ as in (3.3). This is essentially the same result that was obtained by Lüscher for mass corrections which periodic boundary conditions in finite volume [20]. For the case of periodic boundary conditions such corrections to finite volume scattering states and form factors were recently investigated in [21].

Note that the determinant (3.5) has the following behaviour for large $L$ :

$$
\rho_{a_{1} \ldots a_{n}}\left(\tilde{\theta}_{1}, \ldots, \tilde{\theta}_{n}\right)=\left(\prod_{l=1}^{n} 2 m_{a_{l}} L \cosh \tilde{\theta}_{l}\right)\left(1+O\left(L^{-1}\right)\right)
$$

The leading terms is just the density of states for non-interacting particles, and it is independent of the bulk and boundary phase-shifts. The corrections are of the order of $1 /(\mathrm{mL})$ where $m$ is the typical mass scale. In the numerical examples given in section 5 this means that the interaction corrections are of relative size $\sim 10^{-1}$ in the volume range considered there. 


\subsection{Diagonal matrix elements}

When disconnected contributions are present, a more careful analysis is required. As in [7] we must start by finding out the conditions under which there can be equal rapidities in the two states. It is easy to see that due to the finite size corrections coming from the Bethe-Yang equations (3.2) and the positivity of all the quantum numbers $I_{j}$ this can only happen when the quantum number sets happen to be identical, i.e. for the case of diagonal matrix elements

$$
a_{1} \ldots a_{n}\left\langle\left\{I_{1}, \ldots, I_{n}\right\}|\mathcal{O}(0)|\left\{I_{1}, \ldots, I_{n}\right\}\right\rangle_{a_{1} \ldots a_{n}, L}
$$

According to (3.6) for this case we have to consider

$$
F_{\bar{a}_{n} \ldots \bar{a}_{1} a_{1} \ldots a_{n}}\left(\theta_{n}+i \pi, \ldots, \theta_{1}+i \pi, \theta_{1}, \ldots, \theta_{n}\right)
$$

Due to the existence of kinematical poles (2.5) the above expression is not well-defined. Let us consider the regularized version

$$
F_{\bar{a}_{n} \ldots \bar{a}_{1} a_{1} \ldots a_{n}}\left(\theta_{n}+i \pi+\epsilon_{n}, \ldots, \theta_{1}+i \pi+\epsilon_{1}, \theta_{1}, \ldots, \theta_{n}\right)
$$

Just as for bulk form factors, the singular parts of this expression drop when taking the limits $\epsilon_{i} \rightarrow 0$ simultaneously ${ }^{3}$; however, the end result depends on the direction of the limit, i.e. on the ratio of the $\epsilon_{i}$ parameters. The terms that are relevant in the limit can be written in the following general form:

$$
\begin{gathered}
F_{\bar{a}_{n} \ldots \bar{a}_{1} a_{1} \ldots a_{n}}\left(\theta_{n}+i \pi+\epsilon_{n}, \ldots, \theta_{1}+i \pi+\epsilon_{1}, \theta_{1}, \ldots, \theta_{n}\right)= \\
\prod_{i=1}^{n} \frac{1}{\epsilon_{i}} \cdot \sum_{i_{1}=1}^{n} \ldots \sum_{i_{n}=1}^{n} \mathcal{A}_{i_{1} \ldots i_{n}}^{a_{1} \ldots a_{n}}\left(\theta_{1}, \ldots, \theta_{n}\right) \epsilon_{i_{1}} \epsilon_{i_{2}} \ldots \epsilon_{i_{n}}+\ldots
\end{gathered}
$$

where $\mathcal{A}_{i_{1} \ldots i_{n}}^{a_{1} \ldots a_{n}}$ is a completely symmetric tensor of rank $n$ in the indices $i_{1}, \ldots, i_{n}$, and the ellipsis denote terms that vanish when taking $\epsilon_{i} \rightarrow 0$ simultaneously. This tensor can in principle be calculated using an appropriate version the graphical methods developed in [7], but we do not go into details here as it is not needed in the sequel (the interested reader is referred to $[22])$.

The connected matrix element can be defined as the $\epsilon_{i}$ independent part of eqn. (3.7), i.e. the part which does not diverge whenever any of the $\epsilon_{i}$ is taken to zero:

$$
F_{a_{1} \ldots a_{n}}^{c}\left(\theta_{1}, \theta_{2}, \ldots, \theta_{n}\right)=n ! \mathcal{A}_{1 \ldots n}^{a_{1} \ldots a_{n}}\left(\theta_{1}, \ldots, \theta_{n}\right)
$$

where the appearance of the factor $n$ ! is simply due to the permutations of the $\epsilon_{i}$.

For the case of periodic boundary conditions Saleur proposed a way of expressing a diagonal matrix element in terms of connected form factors [23], on the basis of earlier ideas by Balog [24] and the Gaudin determinant formula for the inner product of Bethe states

\footnotetext{
${ }^{3}$ This can be proved by elementary manipulations involving the bulk kinematical singularity axiom $(2.5)$.
} 
[25]. This proposal was tested in [7] for periodic boundary conditions against truncated conformal space, and a spectacular agreement was found.

Saleur's argument actually seems to be quite general, and so the appropriate version of his formula for the boundary case can easily be written, yielding the following conjecture:

$$
\begin{aligned}
& \frac{a_{1} \ldots a_{n}\left\langle\left\{I_{1} \ldots I_{n}\right\}|\mathcal{O}(0)|\left\{I_{1} \ldots I_{n}\right\}\right\rangle_{a_{1} \ldots a_{n}, L}=}{\rho_{a_{1} \ldots a_{n}}\left(\tilde{\theta}_{1}, \ldots, \tilde{\theta}_{n}\right)} \sum_{A \subset\{1,2, \ldots n\}} F_{a(A)}^{c}\left(\left\{\tilde{\theta}_{k}\right\}_{k \in A}\right) \tilde{\rho}_{a_{1} \ldots a_{n}}\left(\tilde{\theta}_{1}, \ldots, \tilde{\theta}_{n} \mid A\right)+O\left(\mathrm{e}^{-\mu L}\right)
\end{aligned}
$$

The summation runs over all subsets $A$ of $\{1,2, \ldots n\}$. For any such subset, we define the corresponding species index set

$$
a(A)=\left\{a_{k}\right\}_{k \in A}
$$

and the appropriate sub-determinant

$$
\tilde{\rho}_{a_{1} \ldots a_{n}}\left(\tilde{\theta}_{1}, \ldots, \tilde{\theta}_{n} \mid A\right)=\operatorname{det} \mathcal{J}_{A}^{a_{1} \ldots a_{n}}\left(\tilde{\theta}_{1}, \ldots, \tilde{\theta}_{n}\right)
$$

of the $n \times n$ Bethe-Yang Jacobi matrix

$$
\mathcal{J}_{a_{1} \ldots a_{n}}\left(\tilde{\theta}_{1}, \ldots, \tilde{\theta}_{n}\right)_{k l}=\frac{\partial Q_{k}\left(\theta_{1}, \ldots, \theta_{n}\right)_{a_{1} \ldots a_{n}}}{\partial \theta_{l}}
$$

obtained by deleting the rows and columns corresponding to the subset of indices $A$. The determinant of the empty sub-matrix (i.e. when $A=\{1,2, \ldots n\}$ ) is defined to equal 1 by convention.

There is a simple way to assign an intuitive meaning to all the terms in (3.9). One needs to consider all possible ways to partition the particles into two sets, one of which $(A)$ is connected to the local boundary operator $\mathcal{O}$, while the particles in the complementary set $\left(A^{\dagger}\right)$ are just contracted with each other using the inner product. For the latter contraction we obtain the inner product

$$
a\left(A^{\dagger}\right)\left\langle\left\{I_{k}\right\}_{k \in A^{\dagger}} \mid\left\{I_{k}\right\}_{k \in A^{\dagger}}\right\rangle_{a\left(A^{\dagger}\right), L} \quad, \quad A^{\dagger}=\{1, \ldots, n\} \backslash A
$$

which is given by the appropriate density of states. As before, this density of states is given by the Jacobian of the quantum number - rapidity mapping. However, this must be considered in the presence of the other particles (those in $A$ ) which contribute to the quantization relations for the particles in the set $A^{\dagger}$, and is therefore given by the corresponding sub-determinant of the Jacobian matrix (3.10).

Finally we remark that the result (3.9) can also be expressed with the so-called symmetric evaluation of diagonal matrix elements instead of the connected one as it was done for the bulk case discussed in [7]; however it turns out that in the boundary case it does not lead to the same simplifications as in the bulk (cf. [22]) and therefore we omit these details in the present work. 


\section{Numerical determination of matrix elements}

\subsection{The boundary truncated conformal space approach}

Truncated conformal space approach (TCSA) was developed by Yurov and Zamolodchikov [26], who used it to describe the scaling Lee-Yang model in finite volume with periodic boundary conditions. The boundary extension of TCSA (dubbed BTCSA) was developed by G. Watts and collaborators and first used in [10]. A detailed description of the method can be found in Runkel's PhD thesis [27], which provides a good starting point for developing a numerical algorithm. We implemented the computations using the symbolic algebra software Mathematica.

Here we restrict ourselves to specify our conventions. Following [10] we used a basis for the operator algebra in which all the structure constants are real. To simplify matters we specified the left boundary condition as the identity one (1) and put the $\Phi$ boundary condition to the right end, which is also the position where our boundary field $\varphi$ is located. In such a case the Hilbert space consists of a single $V_{-1 / 5}$ module whose basis vectors we denote by $|i\rangle$. We truncated this space at various levels, the highest truncation taken at level 25. On this finite dimensional space we computed the matrix elements of the Hamiltonian

$$
H=H_{0}+\lambda \int_{0}^{L} \Phi(\tau=0, x) d x+h \varphi_{R}(\tau=0)
$$

We can measure all quantities in units of the bulk particle mass $m$, and introduce the dimensionless volume variable $l=m L$. The dimensionless Hamiltonian matrix can then be written in the form

$$
h_{i j}=\frac{\pi}{l}\left[\left(\Delta_{i}-\frac{c}{24}\right) \delta_{i j}+\kappa^{\prime}\left(\frac{l}{\pi}\right)^{12 / 5}\left(G^{-1} B\right)_{i j}+\chi(b)\left(\frac{l}{\pi}\right)^{6 / 5}\left(G^{-1} B_{R}\right)_{i j}\right]
$$

where $\Delta_{i}$ is the conformal weight of the basis vector $|i\rangle, c=-22 / 5$ is the central charge, the mass gap constant

$$
\kappa^{\prime}=\kappa^{-12 / 5}=0.097048456298 \ldots
$$

is calculated from (2.14),

$$
\chi(b)=h_{c r i t} \sin \left(\left(b+\frac{1}{2}\right) \frac{\pi}{5}\right)
$$

is the boundary coupling $h(b)(2.13)$ in dimensionless form, $G_{i j}=\langle i \mid j\rangle$ is the usual conformal metric on the state space, and the matrix elements of the perturbing operators

$$
B_{i j}=\left\langle i\left|\int_{0}^{\vartheta} d \vartheta \Phi\left(\mathrm{e}^{i \vartheta}\right)\right| j\right\rangle \quad, \quad\left(B_{R}\right)_{i j}=\langle i|\varphi(1)| j\rangle
$$

are calculated on the upper half plane after the usual exponential mapping from the strip as in [10]. 


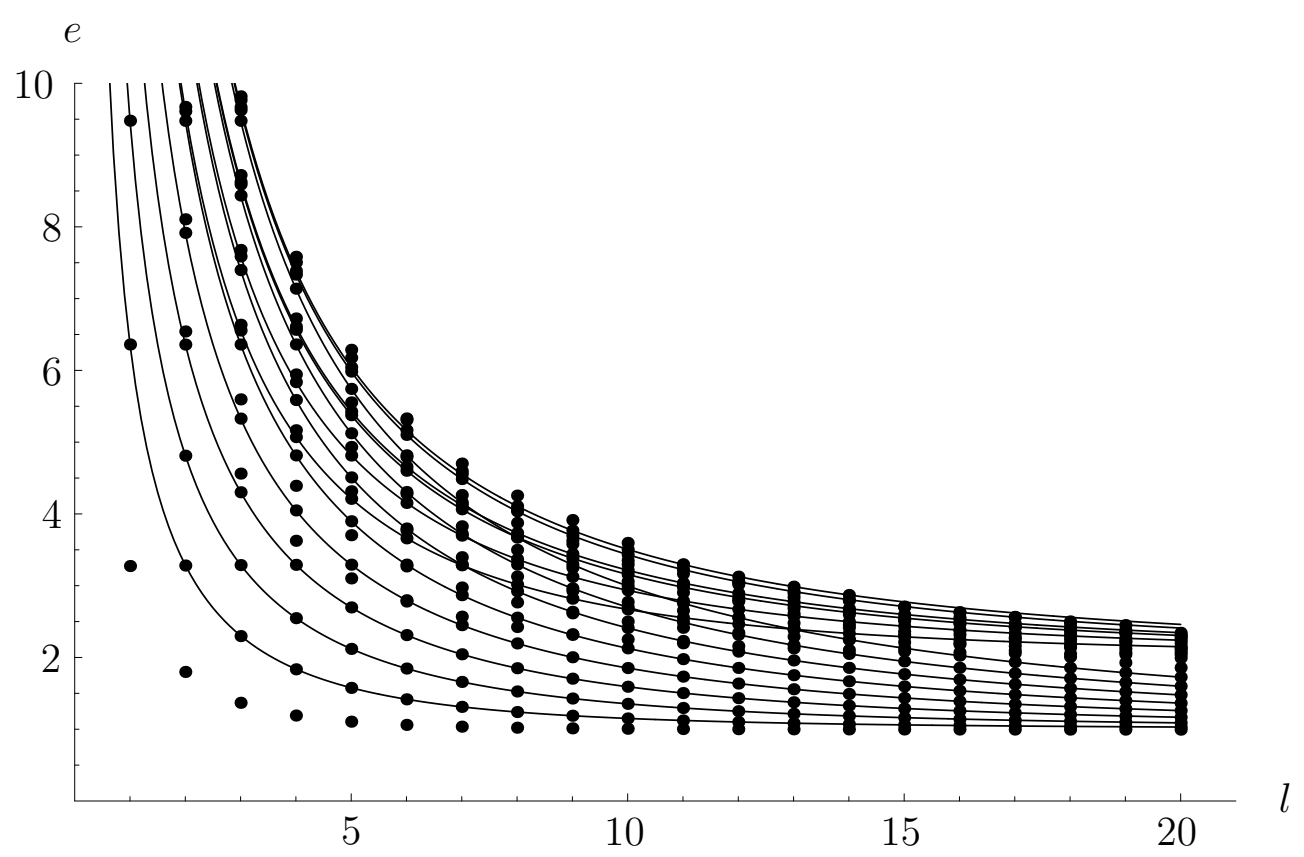

Figure 4.1: BTCSA levels (at truncation level 25) and Bethe-Yang predictions for $b=-0.8$. Energy and volume are measured in units of the bulk particle mass $m$ (i.e. $e=E / m$ and $l=m L$ ). The discrete points are the BTCSA levels (with the ground state subtracted), while the continuous lines are the Bethe-Yang predictions.

\subsection{State identification and evaluation of matrix elements}

Once the BTCSA spectrum is obtained it is just a set of energy levels represented as a bunch of numerical eigenvalues for a series of discreet values of the volume parameter $l=m L$. We need to sort these levels into lines parametrized by the volumes, labeled by their multi-particle contents. This is best achieved by numerical comparison to levels predicted by the Bethe-Yang equations (3.2), which is illustrated in figure 4.1. The figure shows a very good agreement between the two sets of data, however, the lowest state does not fit any of the Bethe-Yang lines. The reason is that it corresponds to a boundary excited state with energy

$$
E_{*}=m \cos \frac{\pi(b+1)}{6}=0.99452 \ldots \times m
$$

Some states corresponding to multi-particle states with the right boundary in this excited state can be seen further up in the spectrum as further points not fitting the Bethe-Yang lines. They can be described if the reflection factor appearing in the Bethe-Yang equations (3.2) is replaced by the one valid for the excited state boundary, but we omit the details here, since the agreement between the BTCSA spectrum and the boundary scattering theory described in section 2.2 was already thoroughly established in [10].

Note that contrary to the case of the periodic TCSA where the Hilbert space can be split into sectors according to total momentum, in BTCSA there is a single sector. 
As a result, the dense part of the spectrum (the "continuum") already starts at the oneparticle threshold, which makes the identification of individual levels harder than it was in the periodic case. We found a number of one-particle and two-particle states, and could also identify the first two three-particle levels over a volume range extending to around $l \sim 14 \ldots 17$, in marked contrast with the periodic case where it was possible to perform a systematic identification of levels with up to 4 particles [6] and even the lowest 5-particle level was found [7]. Even so we could generate a massive amount of useful data for each value of the dimensionless parameter $b$.

Once we identified the levels, we extracted the corresponding eigenvectors and evaluated the matrix elements of the boundary operator $\varphi$ using the method described in [6]. Suppose that we computed two Hamiltonian eigenvectors as functions of the volume $L$ :

$$
\begin{aligned}
\left|\left\{I_{1}, \ldots, I_{n}\right\}\right\rangle_{L} & =\sum_{i} \Psi_{i}\left(I_{1}, \ldots, I_{n} ; L\right)|i\rangle \\
\left|\left\{I_{1}^{\prime}, \ldots, I_{k}^{\prime}\right\}\right\rangle_{L} & =\sum_{j} \Psi_{j}\left(I_{1}^{\prime}, \ldots, I_{k}^{\prime} ; L\right)|j\rangle
\end{aligned}
$$

Let the inner products of these vectors with themselves be given by

$$
\begin{aligned}
\mathcal{N} & =\sum_{i, j} \Psi_{i}\left(I_{1}, \ldots, I_{n} ; L\right) G_{i j} \Psi_{j}\left(I_{1}, \ldots, I_{n} ; L\right) \\
\mathcal{N}^{\prime} & =\sum_{i, j} \Psi_{i}\left(I_{1}^{\prime}, \ldots, I_{k}^{\prime} ; L\right) G_{i j} \Psi_{j}\left(I_{1}^{\prime}, \ldots, I_{k}^{\prime} ; L\right)
\end{aligned}
$$

where $G_{i j}=\langle i \mid j\rangle$ is the metric on the space of the conformal state vectors $|i\rangle$ as before. Then the matrix elements of the field $\varphi$ can be computed as

$$
\begin{aligned}
& m^{1 / 5}\left\langle\left\{I_{1}^{\prime}, \ldots, I_{k}^{\prime}\right\}|\varphi(0)|\left\{I_{1}, \ldots, I_{n}\right\}\right\rangle_{L}= \\
& \quad\left(\frac{\pi}{l}\right)^{-1 / 5} \frac{1}{\sqrt{\mathcal{N}}} \frac{1}{\sqrt{\mathcal{N}^{\prime}}} \sum_{j, l} \Psi_{j}\left(I_{1}^{\prime}, \ldots, I_{k}^{\prime} ; L\right)\left(B_{R}\right)_{j l} \Psi_{l}\left(I_{1}, \ldots, I_{n} ; L\right)
\end{aligned}
$$

where $B_{R}$ is the boundary perturbation matrix entering the boundary Hamiltonian (4.1), and the volume dependent prefactor comes from the transformation of the primary field $\varphi$ under the exponential map. The results will be reported in section 5 , but before that we turn to the estimation of the accuracy of the BTCSA method.

\subsection{Numerical accuracy of BTCSA and error sources}

In order to understand what constitutes a satisfactory agreement between the theoretical predictions and the numerical data, we need to understand the possible sources of deviations.

The truncation inherent in BTCSA introduces a specific source of error, called the truncation error. It grows with the volume $L$ and also it becomes larger when higher 
levels are considered. It is very hard to control and estimate this error very precisely. It can be made smaller by extrapolation procedures such as the one used in [6, 7]. Recently a very efficient approach was introduced in the form of a renormalization group under the variation of the truncation level [28]. To avoid excessive numerical computations, we do not use any extrapolation in the truncation level here; it turns out that the highest truncation level we used (25, which gives a 434 dimensional Hilbert space) is enough to achieve sufficient precision provided we choose the parameter $b$ in a suitable range. We remark that increasing the truncation level to the values used in the periodic case $[6,7]$ (i.e. up to 30) is not very practical because the evaluation of the matrix elements of the bulk perturbation is very time consuming and the required CPU time grows very fast.

Another source of error comes from the fact that numerical diagonalization becomes unstable where levels are nearly degenerate ${ }^{4}$; an example of this phenomenon is shown in subsection 5.2.

A further reason for deviation is that every theoretical prediction that we test against BTCSA is exact only up to residual finite size corrections, i.e. contributions that decay exponentially with the volume. As discussed in [6] for any measured quantity there exists a volume range where the truncation errors and the residual finite size corrections are of the same order; this is the so-called scaling regime where the agreement between the theoretical predictions and the numerical results is optimal.

To see what precision can be expected in the scaling regime, we evaluated three quantities characteristic of the ground state. The energy of the ground state in finite volume has the large volume asymptotics

$$
E_{0}(L)=\mathcal{B} m^{2} L+\mathcal{E}_{b} m+O\left(\mathrm{e}^{-\mu L}\right)
$$

where the exact value of the bulk energy constant is [17]

$$
\mathcal{B}=-\frac{\sqrt{3}}{12}
$$

and the boundary energy constant is given by [10]

$$
\mathcal{E}_{b}=\frac{\sqrt{3}-1}{2}+\sin \frac{\pi b}{6}
$$

In addition, the finite volume vacuum expectation value has the asymptotics

$$
\langle 0|\varphi| 0\rangle_{L}=\langle\varphi\rangle+O\left(\mathrm{e}^{-\mu L}\right)
$$

where the exact asymptotic value $\langle\varphi\rangle$ is given by $(2.19)$.

One can extract estimates for these quantities from the scaling regime of BTCSA, which are compared with the predicted values in table 4.1. Besides noting the very good agreement it is useful to pay particular attention to the vacuum expectation value, because it

\footnotetext{
${ }^{4}$ Eigenvectors corresponding to degenerate or nearly degenerate levels are very sensitive to any small perturbation, and thus even a small truncation error can have a disproportionately large effect.
} 


\begin{tabular}{|c|c||c|c||c|c|}
\hline$b$ & $\mathcal{B}(\mathrm{BTCSA})$ & $\mathcal{E}_{b}$ (exact) & $\mathcal{E}_{b}$ (BTCSA) & $m^{1 / 5}\langle\varphi\rangle($ exact $)$ & $m^{1 / 5}\langle\varphi\rangle(\mathrm{BTCSA})$ \\
\hline \hline-2.3 & -0.144750 & -0.567555 & -0.568208 & -1.023503 & -1.043671 \\
\hline-2.0 & -0.144736 & -0.500000 & -0.499934 & -1.034417 & -1.052965 \\
\hline-1.7 & -0.144588 & -0.411121 & -0.411366 & -1.049802 & -1.066242 \\
\hline-1.4 & -0.144472 & -0.303105 & -0.303446 & -1.070303 & -1.083938 \\
\hline-1.1 & -0.144376 & -0.178614 & -0.178994 & -1.096875 & -1.106887 \\
\hline-1.0 & -0.144351 & -0.133975 & -0.134351 & -1.107309 & -1.115903 \\
\hline-0.8 & -0.144305 & -0.040711 & -0.041154 & -1.136265 & -1.130931 \\
\hline-0.5 & -0.144286 & 0.107206 & 0.106815 & -1.174595 & -1.174435 \\
\hline-0.2 & -0.144315 & 0.261497 & 0.261078 & -1.231176 & -1.226541 \\
\hline 0.0 & -0.144372 & 0.366025 & 0.365596 & -1.278610 & -1.270723 \\
\hline 0.3 & -0.144523 & 0.522460 & 0.522032 & -1.370592 & -1.356133 \\
\hline
\end{tabular}

Table 4.1: Boundary energy and vacuum expectation of $\varphi$ : exact predictions compared to BTCSA. The exact value of $\mathcal{B}$ is $-0.144338 \ldots$.

is in fact the simplest (zero-particle) form factor that can be measured. In fact we found that the deviation of the BTCSA determination of the matrix elements from the theoretical predictions $(3.6,3.9)$ follows well the precision of the determination of the vacuum expectation value. We can therefore see that the highest precision can be achieved when $-1.1 \lesssim b \lesssim 0.0$, and so it is in this range that the numerical tests are the most reliable.

\section{Comparing theoretical predictions with BTCSA data}

In this section we present examples of the comparison between the theoretical predictions $(3.6,3.9)$ and BTCSA data. All the results presented below are for $b=-0.8$, the same value of the model's parameter as in figure 4.1, which is in the optimum range for numerical precision. We actually performed the calculations for fourteen different values of $b$ between -2.3 and +0.3 . We isolated the vacuum state, four one-particle, four two-particle and two three-particle states for each value of $b$, calculated matrix elements for all possible pairwise combinations of these states, and found similarly good agreement as presented below. The numerical deviations gradually increase when moving away from the optimum range of $b$. In the optimum range, we found deviations of the order $10^{-3}$ in the scaling regime; for the extremal values, the deviations increased to a few percent for higher levels. There was not a single matrix element in this huge set of data for which any unexpectedly large deviation occurred; therefore we can state that the numerical data are in full agreement with the theoretical predictions.

We also recall (cf. [6]) that the relative phases of different multi-particle states resulting from numerical diagonalization are in general different from the conventions that follow from the form factor axioms in subsection 2.1. Therefore the relations $(3.4,3.6)$ must be understood to hold up to some phase factors, and in the numerical comparisons in figures 5.1 and 5.3 we use the absolute values of the matrix elements. This issue does not arise 
for the diagonal case as any such phase factor drops out from (3.9); in figure 5.5 we only removed a minus sign that appears on both side of this relation after evaluation.

\subsection{Elementary form factors}

Elementary form factors are defined in eqn. (2.1); these are the ones which enter the form factor equations given in subsection 2.1, and all other form factors can be obtained from them by crossing. Comparing such matrix elements against BTCSA provide a test for form factor functions with all their arguments real, according to eqn. (3.4). However, the numerical magnitude of the corresponding finite volume matrix elements decreases very fast with the number of particles. The three-particle matrix elements are already too small to be measured meaningfully from BTCSA (they are of the order of truncation errors), and therefore we limited ourselves to the one- and two-particle cases, shown in figure 5.1. Note that the two-particle matrix elements are already smaller than the one-particle ones by more than an order of magnitude; accordingly, the relative precision in their case is around one percent, while for the one-particle matrix elements we could achieve around $10^{-3}$ (or even slightly better) in the scaling regime. According to the discussion at the end of subsection 3.2 this is enough to test that the inclusion of the phase-shifts in (3.5) which describe the interaction between the particles and also with the boundary is necessary to achieve agreement between the theoretical predictions (3.4) and truncated conformal space data. This is illustrated in figure 5.2 where besides plotting the correct prediction (3.4) involving the full one-particle density of states

$$
\rho_{1}(\theta)=2 m L \cosh \theta+\frac{\partial \delta^{(\alpha)}(\theta)}{\partial \theta}+\frac{\partial \delta^{(\beta)}(\theta)}{\partial \theta}
$$

which takes into account interactions as defined in (3.5), we also plot a version where the density of states is substituted by

$$
\rho_{1}^{\text {naive }}(\theta)=2 m L \cosh \theta
$$

Here $\delta^{(\alpha)}$ and $\delta^{(\beta)}$ are the boundary phase-shifts define in (3.1). Note that for small $L$ the difference between the naive and the full phase-shift goes to 0 . This may seem surprising at first, but it is due to the fact that $\theta$ increases with decreasing $L$ and the phase-shift derivatives decrease exponentally for $\theta$ away from 0 . Although figure 5.2 only shows the case of the first one-particle state, similar results are obtained for all the form factors discussed in this paper.

\subsection{Non-diagonal matrix elements}

Form factor functions with more than two particles can only be measured with sufficient accuracy from more general (non-diagonal) matrix elements, for which the theoretical prediction is given by eqn. (3.6). We present such data in figure 5.3, which provide a check for form factor functions involving up to 6 particles; the relative deviation between the BTCSA 


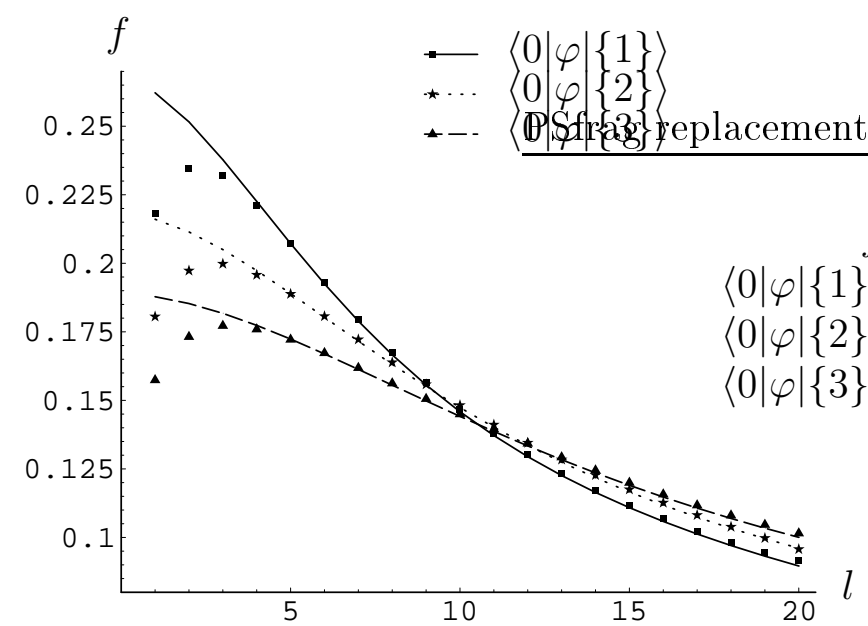

(a) One-particle form factor

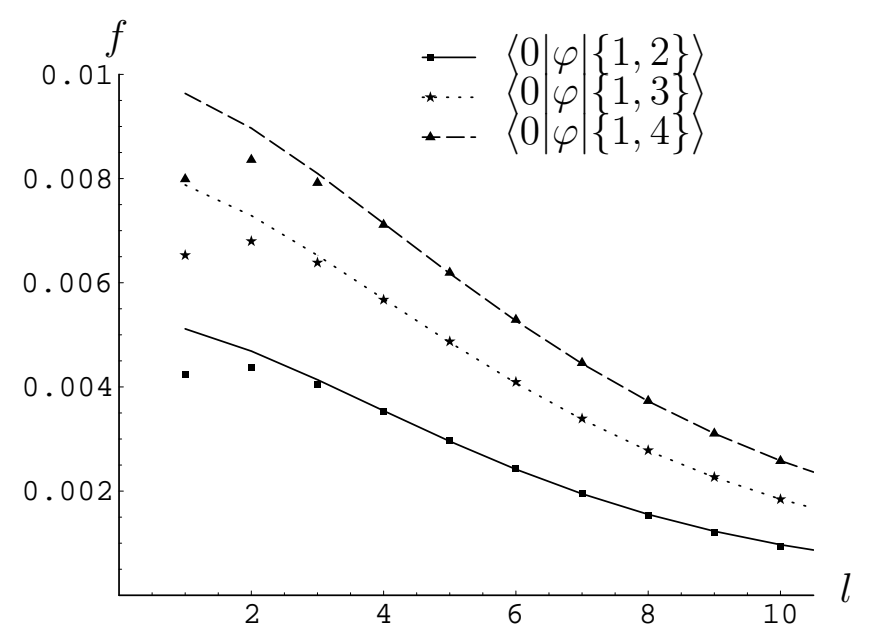

(b) Two-particle form factor

Figure 5.1: One-particle and two-particle elementary form factors. $l=m L$ is the dimensionless volume parameter, while $f$ is the magnitude of the matrix element in units of $m^{-1 / 5}$.

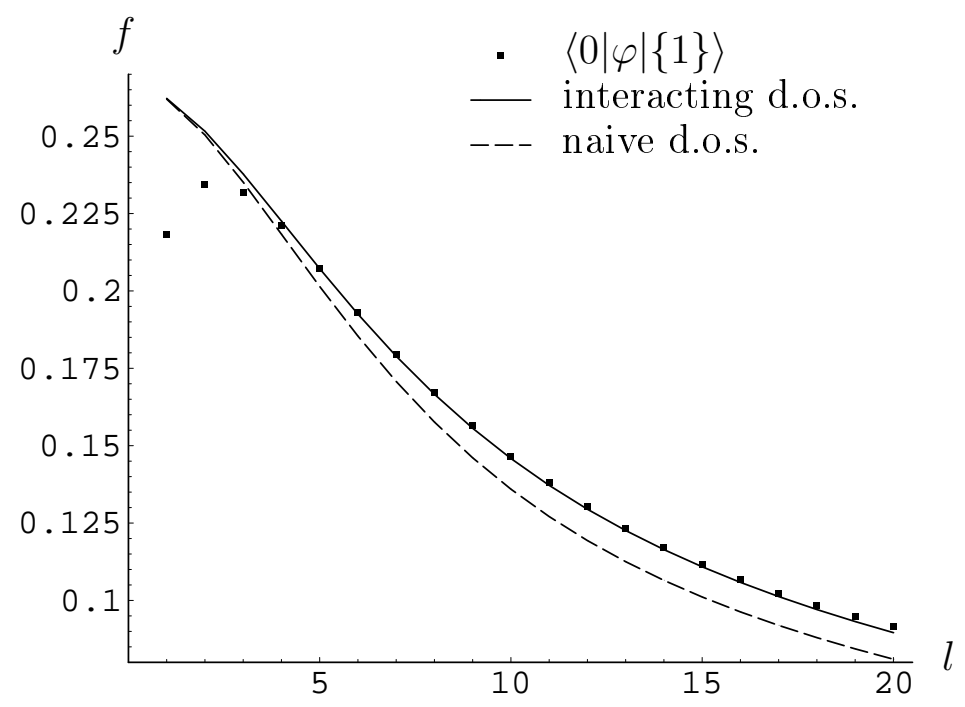

Figure 5.2: The relevance of interaction corrections to the density of states. The discrete points are TCSA data for the one-particle matrix element shown, continous line is the prediction (3.4), while the dashed line shows the same with the naive density of states. 
data and the theoretical prediction (3.6) is around $10^{-3}$ in the scaling regime. We omitted the case of 1-particle-1-particle matrix elements, because they test the two-particle form factor already checked above; they show similarly good agreement. We also omitted the case of 1-particle-3-particle matrix elements as they are again too small to be extracted with high enough precision.

We remark that there is an easily visible, quite large deviation in figure 5.3 (c) at $l=13$. The reason is that the corresponding matrix element involves the second threeparticle level $|\{1,2,4\}\rangle$, which crosses another level in the vicinity. As we already discussed at the beginning of subsection 4.2, in the vicinity of such level crossings the pair of levels can bifurcate into a pair of complex conjugate eigenvalues, which increases the numerical deviation due to truncation errors.

This particular level crossing is shown in figure 5.4, and it can be easily seen that the bifurcation is indeed due to truncation errors. At such a large volume the Bethe-Yang equations (3.2) give a very accurate description of energy levels, since the residual finite size corrections are very small, of order $\mathrm{e}^{-l} \sim 10^{-6}$. Therefore imaginary parts of levels which are larger than this order of magnitude can only be due to truncation errors. Indeed the imaginary parts at the middle of the level crossing are of order $10^{-4}$ which is also consistent with the magnitude of truncation errors that can be estimated from the deviation between the Bethe-Yang and BTCSA levels around this point.

Turning to an exact description of the finite size spectrum, for periodic boundary conditions it can be proved that the full exact finite volume spectrum of the scaling LeeYang model is strictly real [29], and therefore the similar lacunae observed by Yurov and Zamolodchikov [26] can only be due to truncation. We expect that a similar result holds for the scaling Lee-Yang model with boundaries when $b$ is real i.e. when $|h|<h_{\text {crit }}{ }^{5}$, and a proof can probably be given based on the boundary TBA description of the finite volume spectrum established in [10], but we do not pursue this issue further here.

\subsection{Diagonal matrix elements}

The comparisons in the previous two subsections can be considered as a direct verification of the form factor functions given in subsection 2.3. Once we are certain that the elementary form factor functions are correct, we can view the comparison of diagonal matrix elements as testing the structure of the disconnected terms involved in (3.9). The data are presented in figure 5.5 and again show excellent agreement (with deviations of order $10^{-3}$ in the scaling regime) apart from the presence of the deviation due to the level crossing discussed in the previous subsection.

\footnotetext{
${ }^{5}$ For larger values of the boundary coupling $h$ the ground state is destabilized and the spectrum turns complex [10].
} 


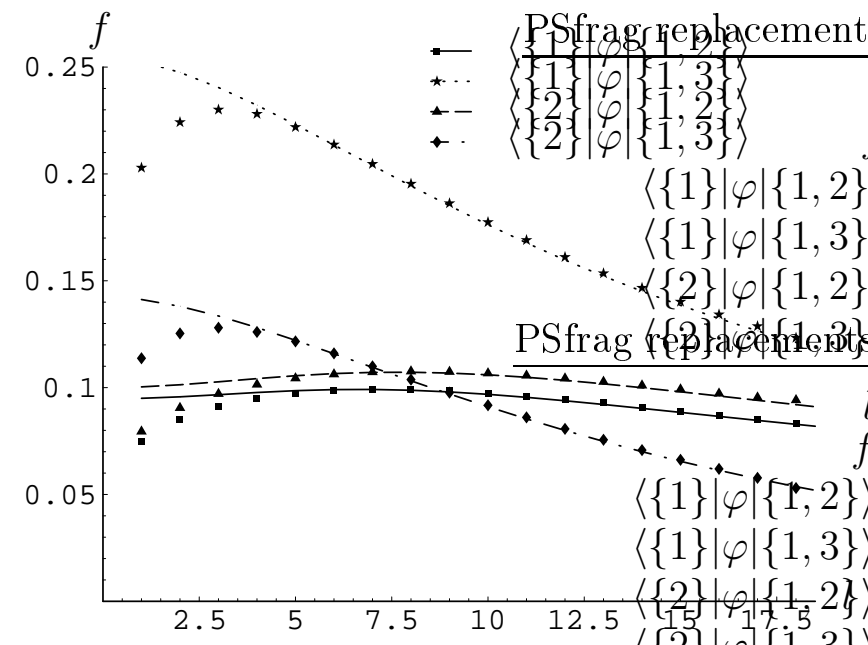

(a) 1-particle-2-particle

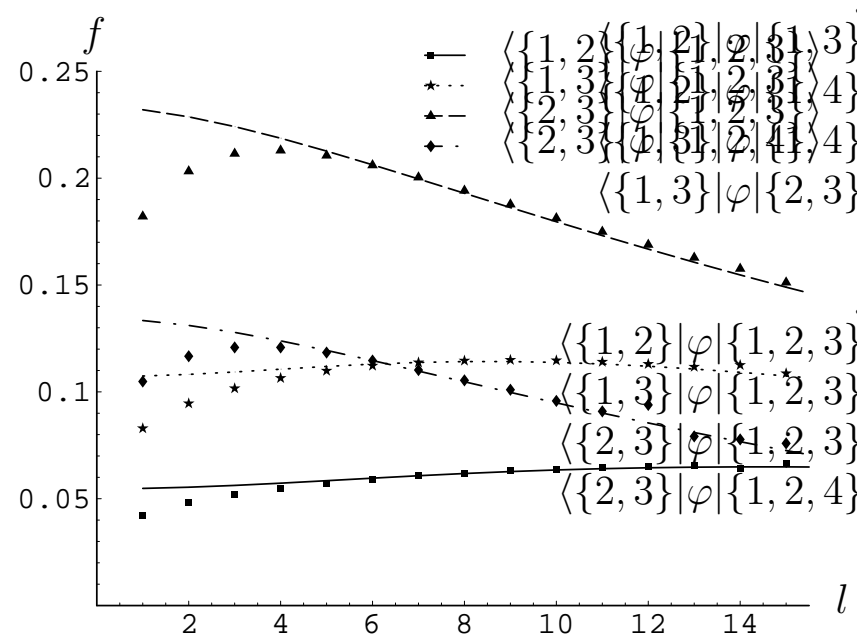

(c) 2-particle-3-particle

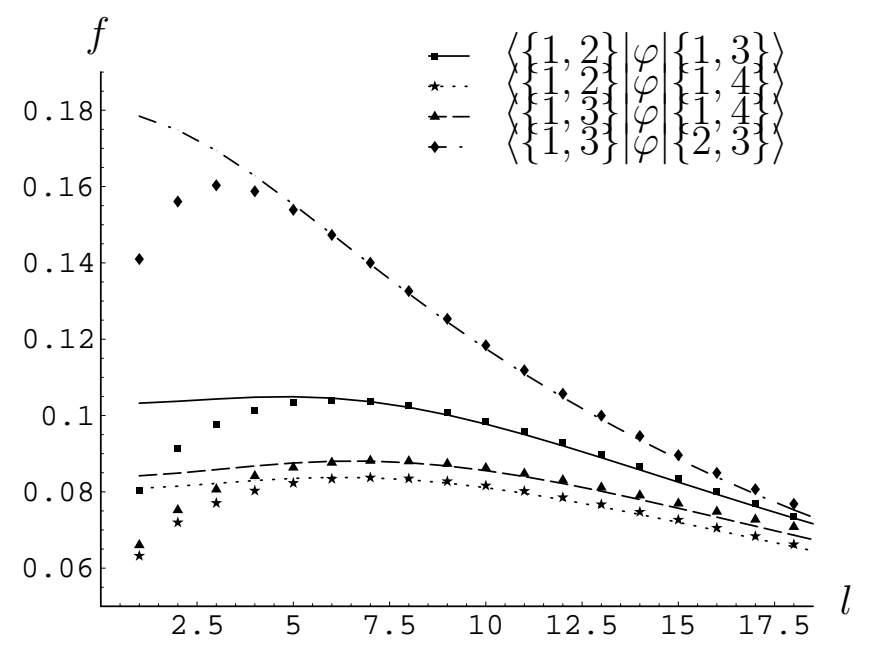

(b) 2-particle-2-particle

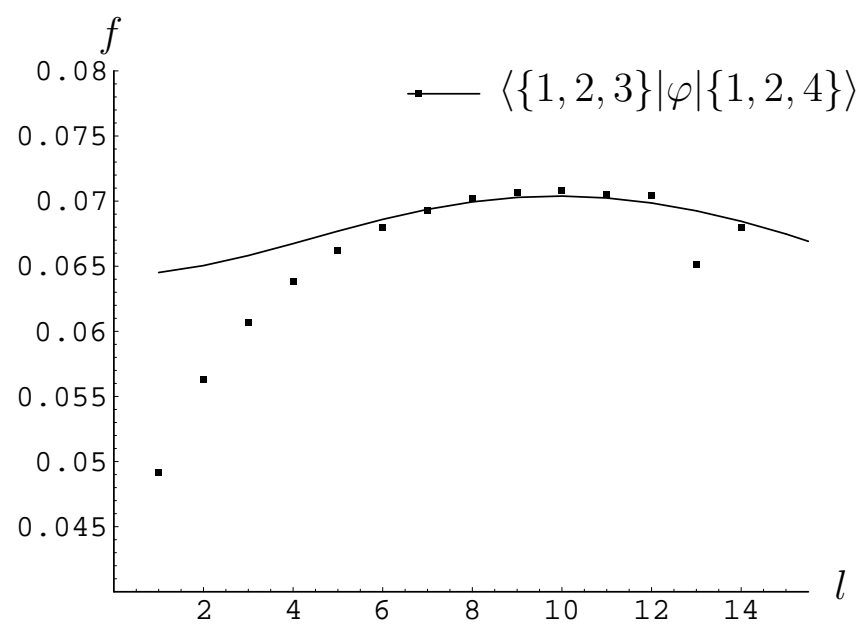

(d) 3-particle-3-particle

Figure 5.3: Non-diagonal matrix elements. $l=m L$ is the dimensionless volume parameter, while $f$ is the magnitude of the matrix element in units of $m^{-1 / 5}$. 

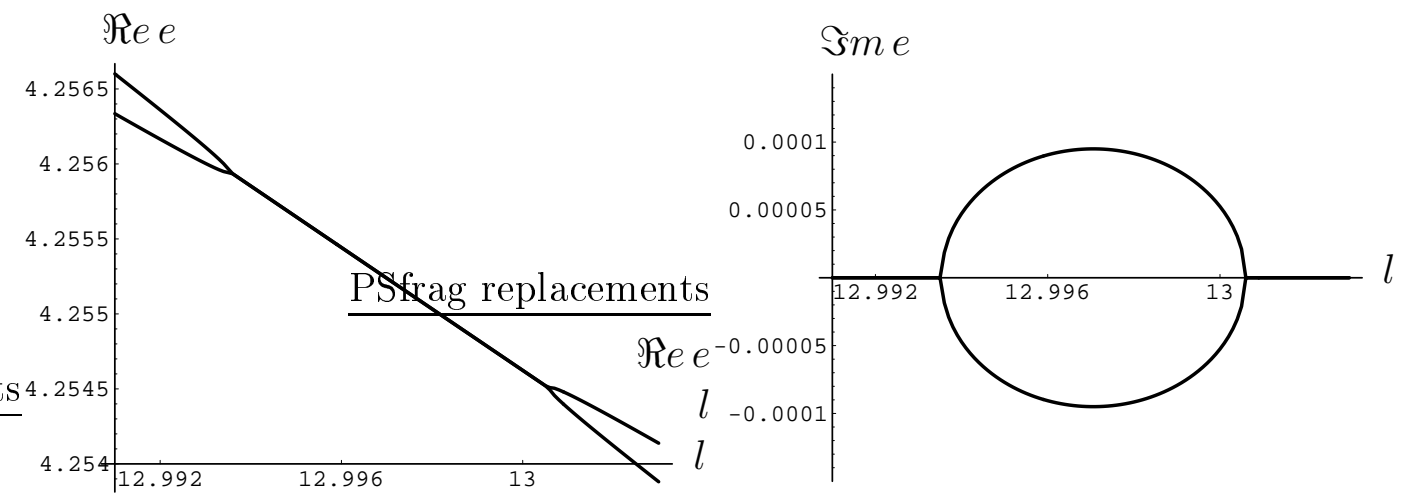

Figure 5.4: Level crossing around $l=13$. Energy $(e)$ is measured in units of $m$, relative to the ground state. The two figures show the real and the imaginary parts of the two levels, respectively.

\section{Conclusions}

In this paper we have succeeded to extend the description of finite volume matrix elements of local operators $[6,7]$ to the case of boundary operators in a boundary quantum field theory. We have compared the predictions of this framework to data extracted from the boundary truncated conformal space approach, and found excellent agreement. This provides a very strong evidence both for the validity of the boundary form factor bootstrap introduced in [2], and to the description of finite volume matrix elements in terms of the infinite volume form factors given by eqns. (3.6,3.9).

As pointed out in [7], understanding finite size corrections to form factors is not only valuable as a tool to check the form factor bootstrap, but also for the calculation of finite temperature correlators. The introduction of finite volume regularizes the singular terms that come from disconnected pieces, and makes it possible to develop a systematic lowenergy expansion for finite temperature correlators, as demonstrated on the example of one-point functions in [7]. A form factor expansion for the finite temperature expectation values of boundary operators has already been developed in [22]; we also plan to extend these results to two-point correlation functions. It was already demonstrated in the seminal paper by Affleck and Ludwig [30] that the finite temperature setting is essential in studying boundary renormalization group flows, and therefore we expect that such an expansion can be useful in this context.

\section{Acknowledgments}

GT wishes to thank Z. Bajnok and L. Palla for useful discussions. This research was partially supported by the Hungarian research fund OTKA K60040. GT was also supported by a Bolyai János research scholarship. 


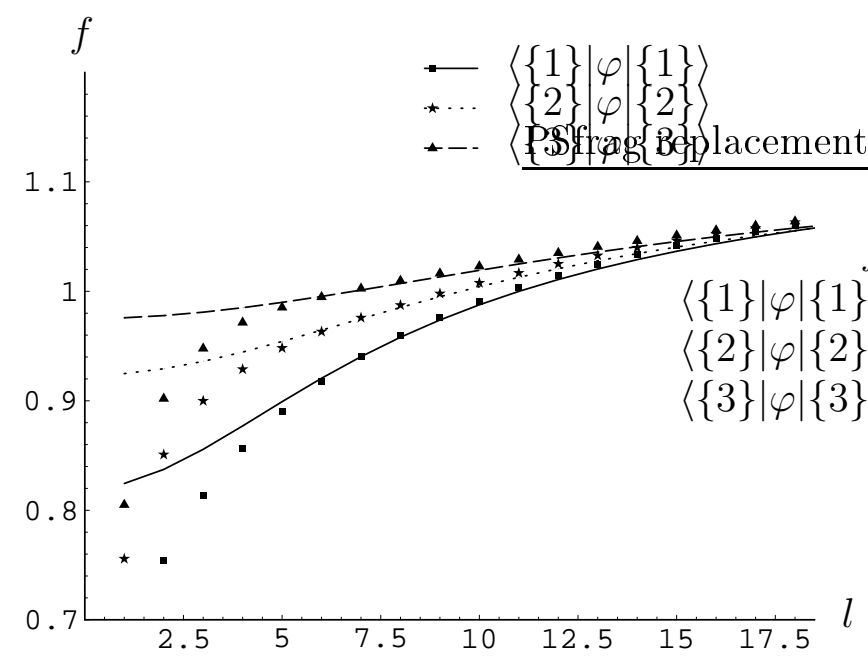

(a) 1-particle

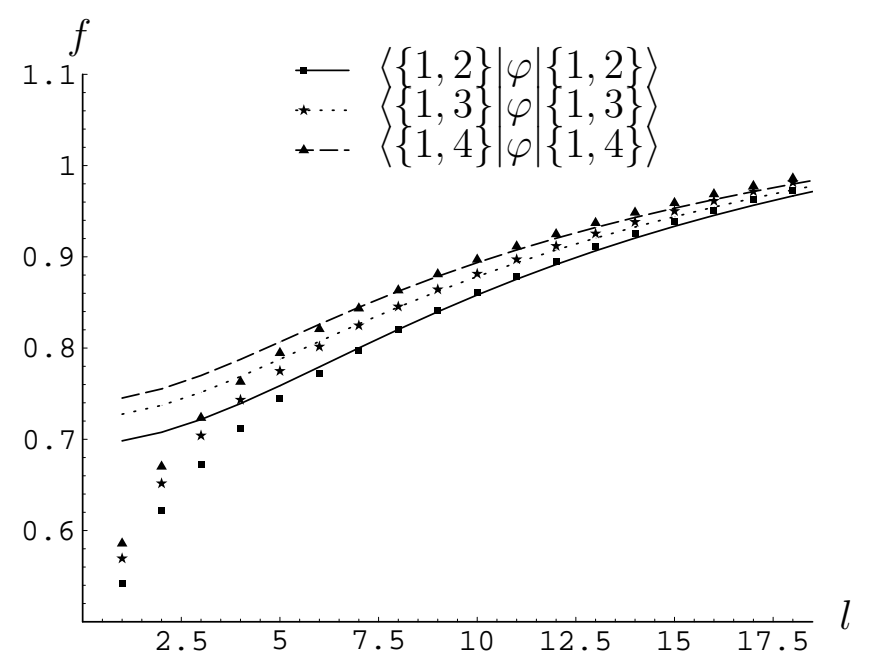

(b) 2-particle

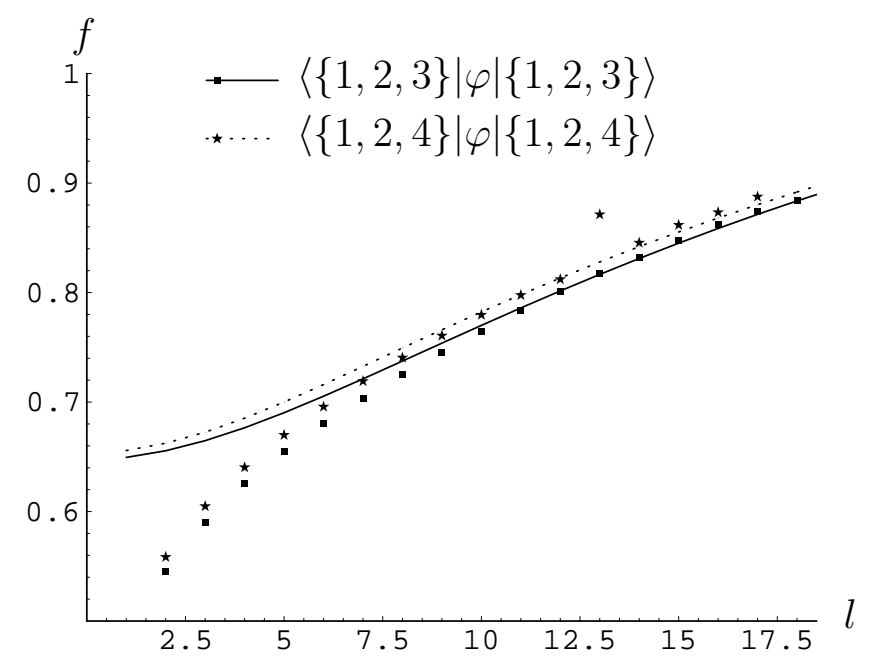

(c) 3-particle

Figure 5.5: Diagonal matrix elements. $l=m L$ is the dimensionless volume parameter, while $f$ is the matrix element in units of $m^{-1 / 5}$, multiplied by -1 (both in the case of the BTCSA data and the theoretical prediction). 


\section{References}

[1] S. Ghoshal and A.B. Zamolodchikov, Int. J. Mod. Phys. A9 (1994) 3841-3886 (Erratum-ibid. A9 4353), hep-th/9306002.

[2] Z. Bajnok, L. Palla and G. Takács, Nucl. Phys. B750 (2006) 179-212, hep-th/0603171.

[3] M. Szôts and G. Takács, Nucl. Phys. B785 (2007) 211-233, hep-th/0703226.

[4] O.A. Castro-Alvaredo, J. Phys. A39 (2006) 11901-11914, hep-th/0606269.

[5] O.A. Castro-Alvaredo: Form factors of boundary fields for A(2)-affine Toda field theory, arXiv:0710.0501 [hep-th].

[6] B. Pozsgay and G. Takács, Nucl. Phys. B788 (2007) 167-208, arXiv: 0706.1445 [hepth].

[7] B. Pozsgay and G. Takács, Nucl. Phys. B788 (2007) 209-251, arXiv: 0706.3605 [hepth].

[8] Z. Bajnok, G. Böhm and G. Takács, J. Phys. A35 (2002) 9333-9342, hep-th/0207079. Z. Bajnok, G. Böhm and G. Takács, Nucl. Phys. B682 (2004) 585-617, hepth/0309119.

[9] M. Karowski and P. Weisz, Nucl. Phys. B139 (1978) 455-476.

[10] P. Dorey, A. Pocklington, R. Tateo and G. Watts, Nucl. Phys. B525 (1998) 641-663.

[11] J.L. Cardy, Nucl. Phys. B324 (1989) 581-596.

[12] J.L. Cardy and D.C. Lewellen, Phys. Lett. B259 (1991) 274-278.

[13] D.C. Lewellen, Nucl. Phys. B372 (1992) 654-682.

[14] J.L. Cardy and G. Mussardo, Phys. Lett. B225 (1989) 275-278.

[15] Al.B. Zamolodchikov, Nucl. Phys. B348 (1991) 619-641.

[16] P. Dorey, I. Runkel, R. Tateo and G. Watts, Nucl. Phys. B578 (2000) 85-122, hepth/9909216.

[17] Al.B. Zamolodchikov, Nucl. Phys. B342 (1990) 695-720.

[18] Al.B. Zamolodchikov, Int. J. Mod. Phys. A10 (1995) 1125-1150.

[19] P. Fendley and H. Saleur, Nucl. Phys. B428 (1994) 681-693, hep-th/9402045.

[20] M. Lüscher, Commun. Math. Phys. 104 (1986) 177. 
[21] B. Pozsgay: Lüscher's $\mu$-term and finite volume bootstrap principle for scattering states and form factors, arXiv:0803.4445 [hep-th].

[22] G. Takács: Finite temperature expectation values of boundary operators, arXiv: 0804.4096 [hep-th].

[23] H. Saleur, Nucl. Phys. B567 (2000) 602-610, hep-th/9909019.

[24] J. Balog, Nucl. Phys. B419 (1994) 480-506.

[25] V.E. Korepin, N.M. Bogoliubov and A.G. Izergin: Quantum inverse scattering method and correlation functions, Cambridge University Press, 1993.

[26] V.P. Yurov and Al.B. Zamolodchikov, Int. J. Mod. Phys. A5 (1990) 3221-3246.

[27] I. Runkel: Boundary problems in Conformal Field Theory, PhD thesis, King's College London, August 2000. Available from http://www.mth.kcl.ac.uk/staff/i_runkel/PDF/phd.pdf.

[28] G. Feverati, K. Graham, P.A. Pearce, G.Zs. Tóth and G. Watts: A Renormalisation group for TCSA, hep-th/0612203. Talk given at the International Workshop on Integrable Models and Applications: From Strings to Condensed Matter (Santiago de Compostela, Spain, 12-16 Sep 2005).

R.M. Konik and Y. Adamov Phys. Rev. Lett. 98 (2007) 147205, cond-mat/0701605.

[29] G. Takács and G.M.T. Watts, Nucl. Phys. B642 (2002) 456-482, hep-th/0203073.

[30] I. Affleck and A.W.W. Ludwig, Phys. Rev. Lett. 67 (1991) 161-164. 